\title{
Nocardia brasiliensis
}

National Cancer Institute

\section{Source}

National Cancer Institute. Nocardia brasiliensis. NCI Thesaurus. Code C86613.

A species of aerobic, Gram-positive, rod shaped bacteria assigned to the phylum Actinobacteria. This species is nonmotile, catalase and urease positive, acid-fast, oxidase negative, hydrolyzes casein, elastin, esculin, hypoxanthine, testosterone, and tyrosine, but not xanthine, produces acid phosphatase, and reduces nitrate. N. brasiliensis is naturally found in soil and is one of the main causative agents of nocardiosis. 